CERN-TH/97-228

\title{
MASSIVE STRING MODES AND NON-SINGULAR PRE-BIG-BANG COSMOLOGY
}

\author{
Michele Maggior"円 \\ Theory Division, CERN, CH-1211 Geneva 23, Switzerland
}

\begin{abstract}
Perturbative $\alpha^{\prime}$ corrections to the low energy string effective action have been recently found to have a potentially regularizing effect on the singularity of the lowest order pre-big-bang solutions. Whether they actually regularize it, however, cannot be determined working at any finite order in a perturbative expansion in powers of the string constant $\alpha^{\prime}$, because of scheme dependence ambiguities. Physically, these corrections are dominated by the integration over the first few massive string states. Very massive string modes, instead, can have a regularizing effect which is non-perturbative in $\alpha^{\prime}$ and which basically comes from the fact that in a gravitational field with Hubble constant $H$ they are produced with an effective Hawking temperature $T=H /(2 \pi)$, and an infinite production rate would occur if this temperature exceeded the Hagedorn temperature. We discuss technical and conceptual difficulties of this non-perturbative regularization mechanism.
\end{abstract}

CERN-TH/97-228

August 1997

revised April 1998

\footnotetext{
${ }^{1}$ Permanent address: INFN and Dipartimento di Fisica, piazza Torricelli 2, I-56100 Pisa, Italy.
} 


\section{Introduction}

String theory is an appropriate framework for discussing the singularities of general relativity and in particular the big-bang singularity, and a large number of works have been devoted to the study of cosmological solutions of the low energy effective action of string theory (see e.g. [1-5]). At lowest order in the string constant $\alpha^{\prime}$, the solutions of the equations of motion still reach a singularity at a finite value of time, say $t=0$, when we evolve the present state of the Universe backward in time [2]. This is not surprising since, in the lowest order effective action, $\alpha^{\prime}$ only enters as an overall constant and therefore drops from the equations of motion; therefore, there is no scale at which the singularity can be regularized. For homogeneous fields, the low energy action has a symmetry, scale factor duality, which relates different solutions of the equations of motions [1, 6]. In the simplest case of an isotropic FriedmannRobertson-Walker (FRW) metric with scale factor $a(t)$, and of a vanishing antisymmetric tensor field $B_{\mu \nu}$, it reads

$$
a \rightarrow \frac{1}{a}, \quad \phi \rightarrow \phi-2 d \log a,
$$

where $\phi$ is the dilaton field and $d$ is the number of spatial dimensions. This symmetry can be generalized to a global $O(d, d)$ symmetry in the more general case of non-diagonal metrics and non-vanishing $B_{\mu \nu}$. Since it involves the dilaton field, it is a string symmetry, with no counterpart in Einstein gravity. Combining scale factor duality with time reversal, it is possible to associate with every 'post-big-bang' solution defined for $t>0$ a 'pre-big-bang' solution defined for $t<0$, through the transformation $a(t) \rightarrow 1 / a(-t)$. Of course, at lowest order in $\alpha^{\prime}$, the pre-big-bang solution also runs into a singularity as $t \rightarrow 0^{-}$. It is therefore natural to ask whether the inclusion of corrections allows a smooth transition between the pre-big-bang and the post-big-bang solutions, providing a non-singular cosmological model.

The effective action of string theory has two different types of perturbative corrections: higher orders in $\alpha^{\prime}$, which are genuinely stringy corrections related to the finite extent of the string, and loop corrections, which carry higher powers of $e^{\phi}$. Perturbative $\alpha^{\prime}$ corrections provide a scale for the regularization of the singularity and have been studied in [7], where it has been found that the equations of motion in the case of constant curvature and linear dilaton reduce, at all orders in $\alpha^{\prime}$, to a set of $(d+1)$ algebraic equations in $(d+1)$ unknowns. If these algebraic equations have a real solution, this can act as late time attractor of the lowest order pre-big-bang solution, as has been shown on specific examples at $O\left(\alpha^{\prime}\right)$. The 
singularity is then replaced by a phase of De Sitter inflation with linearly growing dilaton. To complete the transition to the standard radiation-dominated $F R W$ model (the graceful exit problem) $O\left(e^{\phi}\right)$ corrections must play a crucial role [8, 9, 10, 11].

In this paper we examine again the role of $\alpha^{\prime}$ corrections in string cosmology. In sect. 2, after recalling the results of ref. [0] and extending them to the case of compact dimensions (sect. 2.1), we discuss in some detail the scheme dependence of the results (sect. 2.2) and the relation with scale factor duality (sect. 2.3). Unfortunately, to verify that the mechanism proposed in [7] does take place would require the knowledge of a beta function at all orders in $\alpha^{\prime}$. Therefore, in sect. 3 we try to obtain a better understanding of the physics behind the perturbative corrections and of the general mechanism of smoothing of singularities in string theory. We will relate the perturbative $\alpha^{\prime}$ corrections to the integration over the first few excited string states (sect. 3.1); instead, the mechanism of singularity regularization is

related to the exponential growth of the density of states; it is therefore basically due to very massive string modes.

In sect. 4 we study the effect on the lowest order solution of the backreaction due to very massive string modes produced by the gravitational field. For technical reasons, that we will discuss in sect. 4, it turns out to be quite difficult to present an explicit non-singular solution. The result also suggests that treating massive modes production as a backreaction, in a full stringy regime, is not adequate. However, from the equations that include the creation of massive modes, we will be able to obtain at least a qualitative understanding of the role of massive modes production and of their possible regularizing effect. In sect. 5 we present the conclusions and we discuss possible phenomenological implications of a string phase with highly excited massive modes.

\section{$2 \quad$ Perturbative $\alpha^{\prime}$ corrections}

\subsection{The constant curvature solution}

Let us recall the results of ref. [7]. Including first order corrections in $\alpha^{\prime}$, a possible form of the effective action, in the string frame, is [12, 13

$$
S=-\frac{1}{2 \lambda_{s}^{d-1}} \int d^{d+1} x \sqrt{-g} e^{-\phi}\left[R+(\nabla \phi)^{2}-\frac{k \alpha^{\prime}}{4} R_{\mu \nu \rho \sigma}^{2}\right],
$$


where $\lambda_{s}$ is the string length, $\lambda_{s} \sim \sqrt{\alpha^{\prime}}, k=1,1 / 2$ for the bosonic and heterotic string, respectively, and we have neglected the antisymmetric tensor field. For type II strings $k=0$, and the first correction starts at order $R_{\mu \nu \rho \sigma}^{4}$ [14. Performing the field redefinition $g_{\mu \nu} \rightarrow g_{\mu \nu}+\left(k \alpha^{\prime}\right) \delta g_{\mu \nu}, \phi \rightarrow \phi+\left(k \alpha^{\prime}\right) \delta \phi$ with

$$
\delta g_{\mu \nu}=R_{\mu \nu}-\partial_{\mu} \phi \partial_{\nu} \phi+g_{\mu \nu}(\nabla \phi)^{2}, \quad \delta \phi=\frac{1}{4}\left[R+(2 d-3)(\nabla \phi)^{2}\right]
$$

and truncating at first order in $\alpha^{\prime}$ gives the action

$$
S=-\frac{1}{2 \lambda_{s}^{d-1}} \int d^{d+1} x \sqrt{-g} e^{-\phi}\left[R+(\nabla \phi)^{2}-\frac{k \alpha^{\prime}}{4}\left(R_{G B}^{2}-(\nabla \phi)^{4}\right)\right],
$$

where $R_{G B}^{2}=R_{\mu \nu \rho \sigma}^{2}-4 R_{\mu \nu}^{2}+R^{2}$ is the Gauss-Bonnet term. This form is particularly convenient because higher order derivatives in the $\alpha^{\prime}$ correction cancel after integrations by part [15]. We will discuss in some detail field redefinitions in sect. 2.2.

We now restrict ourselves to a homogeneous and isotropic $F R W$ background, $d s^{2}=$ $N^{2}(t) d t^{2}-a^{2}(t) d \mathbf{x}^{2}$ and homogeneous dilaton $\phi=\phi(t)$. Varying the action with respect to $a, \phi$, we get two dynamical equations of motion. Introducing $H=\dot{a} / a$ and specializing to $d=3$ for notational simplicity, they read (in units $k \alpha^{\prime}=1$ ):

$$
\begin{gathered}
-6 \dot{H}\left(1+H^{2}\right)+2 \ddot{\phi}\left(1+\frac{3}{2} \dot{\phi}^{2}\right)-12 H^{2}+6 H \dot{\phi}-\dot{\phi}^{2}-\frac{3}{4} \dot{\phi}^{4}-6 H^{4}+3 H \dot{\phi}^{3}=0 \\
-12 \dot{H}(1-H \dot{\phi})+6 \ddot{\phi}\left(1+H^{2}\right)-18 H^{2}+12 H \dot{\phi}-3 \dot{\phi}^{2}+\frac{3}{4} \dot{\phi}^{4}+12 H^{3} \dot{\phi}-6 H^{2} \dot{\phi}^{2}=0 .
\end{gathered}
$$

The variation with respect to the lapse function $N$ gives, instead, a constraint on the initial values:

$$
6 H^{2}-6 H \dot{\phi}+\dot{\phi}^{2}-6 H^{3} \dot{\phi}+\frac{3}{4} \dot{\phi}^{4}=0 .
$$

The constraint is conserved by the dynamical equations of motion, and is therefore satisfied at any time if it is satisfied at the initial time. We can now try to solve eqs. 2.4 2.6) with the ansatz $H=$ const $=y, \dot{\phi}=$ const $=x$. The ansatz reduces the three differential equations to algebraic equations in $x, y$. At first sight we have three independent equations for two unknown variables. However, reparametrization invariance gives one relation between these equations such that if the constraint equation and, say, the equation obtained with a variation with respect to $a$ (or, in the general anisotropic case, the $d$ equations obtained with a variation with respect to $\left.a_{i}, i=1, \ldots, d\right)$ are satisfied, then the equation obtained with a variation with respect to $\phi$ is automatically satisfied. 
In the generic anisotropic case with scale factors $a_{i}(i=1, \ldots, d)$, the ansatz therefore reduces the system of $(d+2)$ differential equations to $(d+1)$ algebraic equations in $(d+1)$ variables $H_{i}=y_{i}, \dot{\phi}=x$, and this is true at all orders in $\alpha^{\prime}$.

These algebraic equations are nothing but the requirement that there is a zero in the beta functionals of the underlying sigma model, when the background is specialized to the form of our ansatz, and we will therefore write them as $\beta_{i}(\mathbf{g})=0$, where $i=1, \ldots,(d+1)$ and $\mathbf{g}=\left(x, y_{1}, \ldots, y_{d}\right)$.

It is easy to generalize this result to the case of compact dimensions. Let us first consider the case in which the compact space is spatially curved, e.g. consider four-dimensional space $M^{4}$ times $S^{2}$, with metric

$$
d s^{2}=N^{2}(t) d t^{2}-\sum_{i=1}^{3} a_{i}^{2}(t) d x_{i}^{2}-b^{2}(t)\left(d \theta^{2}+\sin ^{2} \theta d \phi^{2}\right) .
$$

(The extension to more general cases will be obvious.) Writing down the equations of motion we see that now $b$ enters not only in the combination $\dot{b} / b$, but also through terms $\sim 1 / b^{2}$, which are due to the curvature of the sphere. Therefore the ansatz $\dot{a}_{i} / a_{i}=$ constant, $\dot{\phi}=$ constant can only be consistent if $b=$ const, or $\dot{b} / b=0$. Again for the ansatz $\dot{a}_{i} / a_{i}=$ $y_{i}, \dot{\phi}=x, b=$ const the relation between equations of motion derived from reparametrization invariance eliminates one equation and we have a number of algebraic equations equal to the number of variables $y_{i}, x, b$.

The same happens if, instead, we compactify on a torus. In this case the explicit dependence on the scale factor, which forces it to be constant, comes from the winding modes, whose energy grows with the scale factor.

In conclusion, the existence of a solution with $H_{i}, \dot{\phi}$ constant (and $b$ constant, for compact dimensions) depends on whether the algebraic equations discussed above have real solutions. For the action (2.3) this is indeed the case, and the solution turns out to be an attractor of the lowest order pre-big-bang solution, which is therefore regularized [7]. However, the inclusion of higher orders in $\alpha^{\prime}$ or field redefinitions of the type used in eq. (2.2) produce different algebraic equations, which may or may not have real solutions. These issues, which were already noted in ref. [7], will be further discussed in the next section. 


\subsection{Scheme dependence of the results}

In principle, we would like to know the beta functions $\beta_{i}(\mathbf{g})$ exactly, while what we have is a perturbative expansion in powers of $\alpha^{\prime}$. Somewhat optimistically, one might still hope to find a zero which, in units $k \alpha^{\prime}=1$, is located at $g_{i} \ll 1$, thus justifying a perturbative treatment. Unfortunately, an even more fundamental obstacle stands in the way. The problem is that, if we work at finite order in $\alpha^{\prime}$, the coefficients of the algebraic equation, or of the expansion of the functions $\beta_{i}$, are subject to ambiguities. A straightforward way to understand this point is to observe that we can perform fields redefinitions that mix different orders in $\alpha^{\prime}$. The most general form of such redefinitions, at order $\alpha^{\prime}$, is [16] $g_{\mu \nu} \rightarrow g_{\mu \nu}+\left(k \alpha^{\prime}\right) \delta g_{\mu \nu}$, $\phi \rightarrow \phi+\left(k \alpha^{\prime}\right) \delta \phi$, with

$$
\begin{gathered}
\delta g_{\mu \nu}=a_{1} R_{\mu \nu}+a_{2} \partial_{\mu} \phi \partial_{\nu} \phi+a_{3} g_{\mu \nu}(\nabla \phi)^{2}+a_{4} g_{\mu \nu} R+a_{5} g_{\mu \nu} \square \phi \\
\delta \phi=b_{1} R+b_{2}(\nabla \phi)^{2}+b_{3} \square \phi .
\end{gathered}
$$

It is not necessary to include a term $\nabla_{\mu} \partial_{\nu} \phi$ in eq. (2.8) because it can be reabsorbed by a general coordinate transformation [16]. After this redefinition, the new action, truncated at order $\alpha^{\prime}$, is (in units $k \alpha^{\prime}=1$ )

$$
\begin{gathered}
S=-\int d^{d+1} x \sqrt{-g} e^{-\phi}\left[R+(\nabla \phi)^{2}-\frac{1}{4} R_{\mu \nu \rho \sigma}^{2}+c_{1} R_{\mu \nu} R^{\mu \nu}+c_{2} R^{2}+c_{3}(\nabla \phi)^{4}+\right. \\
\left.+c_{4} R^{\mu \nu} \partial_{\mu} \phi \partial_{\nu} \phi+c_{5} R(\nabla \phi)^{2}+c_{6} R \square \phi+c_{7} \square \phi(\nabla \phi)^{2}+c_{8}(\square \phi)^{2}\right],
\end{gathered}
$$

with the coefficients $c_{i}$ functions of $a_{i}, b_{i}$. (We have eliminated terms that can be reduced to the above terms after integrations by part or use of Bianchi identity). Despite the fact that we have eight coefficients $c_{i}$ and eight parameters $a_{1}, \ldots, a_{5}, b_{1}, b_{2}, b_{3}$, we cannot fix the $c_{i}$ at arbitrary values since, from the explicit expression of the $c_{i}$ as functions of $a_{1}, \ldots, b_{3}$, one finds that they satisfy a relation $c_{2}+c_{3}+c_{7}+c_{8}=c_{5}+c_{6}$. Within this constraint, however, the $c_{i}$ can be chosen at will with the appropriate field redefinitions. The existence of a relation between the $c_{i}$ means that there is a one-parameter family of field redefinitions which, at order $\alpha^{\prime}$, leaves the action invariant. It is readily found to be

$$
\begin{gathered}
\delta g_{\mu \nu}=\zeta g_{\mu \nu}\left(R-(\nabla \phi)^{2}+2 \square \phi\right), \\
\delta \phi=\zeta\left(\frac{d-1}{2} R-\frac{d+1}{2}(\nabla \phi)^{2}+d \square \phi\right),
\end{gathered}
$$


with $\zeta$ a real parameter. We now ask whether the existence of a zero of the functions $\beta_{i}$, at a given order in $\alpha^{\prime}$, is affected by the field redefinitions, and the answer is positive. For instance, we can fix $c_{1}=1, c_{2}=-1 / 4, c_{3}=1 / 4$, and vary $c_{4}$ (which does not enter the relation between the $c_{i}$ ) setting all others $c_{i}$ to zero. For $c_{4}=0$ we have the action used in [7], and there is a zero of the beta functions. In $d=3$, for the isotropic case, it is located at $x \simeq 1.404, y \simeq 0.616$. Increasing $c_{4}$ this zero disappears (escaping at infinity in the $(x, y)$ plane) at a critical value $c_{4} \simeq 0.05$.

Therefore, while we expect that, at all orders in $\alpha^{\prime}$, the existence of a zero in the beta functions is independent of field redefinitions, this is not true for the truncation at any finite order in $\alpha^{\prime}$.

There are other useful ways to understand the existence of ambiguities in the perturbative expansion of the functions $\beta_{i}$, and these different points of view are believed to be equivalent. First, from the point of view of the underlying sigma model, they are due to the dependence of the perturbative coefficients of the beta functionals on the renormalization scheme. This dependence starts at two loops, i.e. from the terms $\sim R^{2}$ in the action, eq. (2.10).

The effective low energy action, on the other hand, is constructed in such a way as to reproduce the string theory $S$-matrix elements. From this point of view, the ambiguities in the coefficients come from the fact that some coefficients cancel in the computation of on-shell amplitudes [17, 16, 18]. Suppose for instance that we want to fix the coefficients of the operators $R_{\mu \nu \rho \sigma}^{2}, R_{\mu \nu}^{2}$ and $R^{2}$ in the effective action. We would then expand these operators around the flat metric, $g_{\mu \nu}=\eta_{\mu \nu}+h_{\mu \nu}$ and compute their contribution to the 3point and 4-point amplitudes. However, the $\sim h^{3}$ part of $\sqrt{-g} R_{\mu \nu} R^{\mu \nu}$ and of $\sqrt{-g} R^{2}$ vanish; therefore, the coefficients $c_{1}, c_{2}$ in the action (2.10) cannot be determined from the knowledge of the string amplitude with three on-shell gravitons. Both $\sqrt{-g} R_{\mu \nu} R^{\mu \nu}$ and $\sqrt{-g} R^{2}$ are non-vanishing when expanded at order $h^{4}$. However, the string amplitude includes oneparticle reducible graphs with graviton exchange; to reproduce it we must therefore sum the contact and the exchange terms derived from the effective action. In the sum, the coefficients $c_{1}, c_{2}$ cancel (see 18 for a detailed computation), and so they cannot be determined from the comparison with string amplitudes. The coefficient of $R_{\mu \nu \rho \sigma}^{2}$ is instead fixed by this procedure, at the value $-1 / 4$. 


\subsection{Relation with scale factor duality}

Scheme dependence should not change physical results if we had the exact expression for the beta functions. It enters into play when we truncate at finite order in $\alpha^{\prime}$. When scheme dependence appears, some scheme will be 'better' than others, in the sense that the results obtained at finite order in this scheme will be closer to the exact result. Since scale factor duality plays an important role in motivating the cosmological model that we are discussing, one might hope that a scheme that respects scale factor duality at a given order in $\alpha^{\prime}$ will be better, in the above sense.

Scale factor duality has been generalized to $O\left(\alpha^{\prime}\right)$ in refs. [19, 20]. The analysis of ref. [19] is very general, and refers to the full $O(d, d)$ symmetry. The result of ref. [19] is that there is one, and only one action invariant under scale factor duality at order $\alpha^{\prime}$, and it is given by the action (2.10) with $c_{1}=1, c_{2}=-1 / 4, c_{3}=1 / 4, c_{4}=1, c_{5}=-1 / 2, c_{6}=0, c_{7}=-1 / 2, c_{8}=0$. At the same time, the duality transformation must acquire $\alpha^{\prime}$ corrections. If we restrict to $F R W$ metrics and vanishing antisymmetric tensor field, then $a_{i} \rightarrow 1 / a_{i}\left(\right.$ or $\left.\log a_{i} \rightarrow-\log a_{i}\right)$ must be generalized as

$$
\log a_{i} \rightarrow-\left(\log a_{i}\right)-k \alpha^{\prime}\left(\frac{\dot{a}_{i}}{a_{i}}\right)^{2} .
$$

The transformation of the dilaton is fixed from the condition that $\phi-\sum_{i} \log a_{i}$ is invariant. In $d=3$, for isotropic metric, the equations $\beta_{i}(x, y)=0$ for this dual action have two real solutions at $(x \simeq 1.526, y \simeq 1.913)$ and at $(x \simeq 6.201, y \simeq 1.931)$ (plus the solutions $(-x,-y)$, which are always associated with the solution $(x, y))$. The existence of a pair of solutions (plus the sign-reversed pair) is related to the scale factor duality of the action. However, for the action that we are considering, scale factor duality is valid only at order $\alpha^{\prime}$, and not exactly, since the transformation (2.13), when applied to the $O\left(\alpha^{\prime}\right)$ terms of the action, generates $O\left(\alpha^{\prime 2}\right)$ terms that are truncated. Thus the two solutions are not exactly dual to each other.

Integrating the equations of motion numerically, one finds [7] that these solutions do not act as a late time attractor of the lowest order solution, which still diverges at some finite value of time.

To further explore the relation with duality we have tried a different generalization of

\footnotetext{
${ }^{2}$ Real solutions for this action exist for any $d$. This can be proved numerically up to large values of $d$, where the results matches with a large $d$ expansion.
} 
duality at $O\left(\alpha^{\prime}\right)$. In fact, the action found in 19 and the form of the duality transformation (2.13) are uniquely fixed only if we consider the general case of non-isotropic metrics. If however we restrict to isotropic metrics, a transformation

$$
\log a \rightarrow-(\log a)-\lambda k \alpha^{\prime}\left(\frac{\dot{a}}{a}\right)^{2}
$$

with arbitrary $\lambda$, leaves the action (2.10) invariant at order $\alpha^{\prime}$ if the coefficients $c_{i}$ are $c_{1}=$ $1, c_{2}=-1 / 4, c_{3}=1 / 4+(\lambda-1) / 18, c_{4}=1+(\lambda-1) / 9, c_{5}=-c_{4} / 2, c_{6}=0, c_{7}=-2 c_{3}, c_{8}=0$. (The relation between the $c_{i}$ is still satisfied.) For $\lambda=1$ we recover the action and the transformation of ref. [19]. However, for every transformation (2.14) with given $\lambda$ we now have one action that is invariant. [n a sense, this enlarged family of transformations is less interesting than the transformation found in [19], because the latter is the only one that can be generalized to the anisotropic case and to the inclusion of the antisymmetric tensor field. However, this family of transformations also includes the case $\lambda=0$, i.e. the transformation $a \rightarrow 1 / a$, without $\alpha^{\prime}$ corrections, which is appealing for its simplicity. Furthermore, since this transformation does not generate higher orders in $\alpha^{\prime}$, it is an exact invariance of the action (2.10), with the appropriate choice of $c_{i}$, rather than an invariance at $O\left(\alpha^{\prime}\right)$.

We have therefore studied the equations of motion for the action with $\lambda=0$ duality. We have found that the functions $\beta_{i}(x, y)$ have a pair of zeros (plus the sign reversed pair), and these zeros are related by exact duality invariance, as it should. But, again, these solutions do not act as late time attractors of the lowest order solution, which instead runs into a singularity. The type of singularity is however different from the one encountered for the case $\lambda=1$. It is interesting to understand in some detail what happens. The dynamical equations of motion can be written in matrix form

$$
A\left(\begin{array}{c}
\ddot{\phi} \\
\dot{H}
\end{array}\right)=\left(\begin{array}{c}
f_{1} \\
f_{2}
\end{array}\right)
$$

where $A$ is a $2 \times 2$ matrix that depends on $H, \dot{\phi}$, and $f_{1}, f_{2}$ are functions of $H, \dot{\phi}$. At a finite value of time we find that $\operatorname{det} A=0$, and therefore the equations become singular. As a simpler example of a similar situation, consider the differential equation $(1-h) \dot{h}=h$ for

\footnotetext{
${ }^{3}$ Basically, we have a much less rigid structure because many expressions which are independent in the anisotropic case, such as $\sum_{i} H_{i}^{2}$ and $\left(\sum_{i} H_{i}\right)^{2}$, collapse to the same expression in the isotropic case, and we can arrange cancellations between them.
} 
some function $h(t)$. For small $h$ the solution grows like $e^{t}$, until the 'determinant' $(1-h)$ vanishes, so the situation is similar to our case. Here however we can integrate the equation exactly, and find $t(h)=\log h-h$. Above a critical value $t=-1$, this relation cannot be inverted while, below this value, there are two branches $h_{+}(t)$ and $h_{-}(t)$ that merge at $t=-1$.

This suggests that what happens in our case is that the doubling of solutions due to duality transformations with $\lambda=0$ produces pairs of solutions of the dynamical equations of motion, which merge at some critical value of time, and thereafter move into the complex plane. (These solutions are not dual to each other. One corresponds to a small initial value

of $H$ and $\dot{H}>0$, and the other to $H$ large, and $\dot{H}<0$, while duality changes $H \rightarrow-H)$. If this conjecture is correct, then it appears that duality works against the regularization mechanism. In any case, neither a scheme that respects duality with $\lambda=1$ nor a scheme that respects duality with $\lambda=0$ provides a realization of the regularization mechanism, at $O\left(\alpha^{\prime}\right)$.

\section{The physics behind the regularization of the singu- larity}

The conclusion of the previous sections is that perturbative $\alpha^{\prime}$ corrections can in principle regularize the singularity; however, to determine whether this actually happens we should know the functions $\beta_{i}$ at all orders in $\alpha^{\prime}$, because results at finite order are scheme dependent. The attempt to fix the scheme using scale factor duality as a guiding principle does not give encouraging results. We therefore ask whether there are general physical principles that suggest that the singularity is indeed regularized. Generally speaking, we expect that string theory eliminates all unwanted singularities. However, is the physical mechanism that eliminates the singularity related to perturbative $\alpha^{\prime}$ corrections? Or should we look for a different type of corrections? In this section we discuss this question.

\subsection{Effective action and massive string modes}

As it is well known, the low energy action of string theory can be obtained either by computing the beta functions of the sigma model or from the requirement of reproducing the 
$S$-matrix elements. The two methods are equivalent, as was proved in [21]. Still, the physical pictures underlying the two computations are quite different. In particular, in the first method the massive string modes seem to play no role at all.

In fact, what one does in order to include massive modes at the sigma model level is the following. The sigma model action is written including the interaction with the background fields representing the massive modes [13, 22],

$$
\begin{gathered}
S=\frac{1}{\alpha^{\prime}} \int d^{2} \sigma \sqrt{-h}\left[g_{\mu \nu}(X) h^{i j} \partial_{i} X^{\mu} \partial_{j} X^{\nu}+B_{\mu \nu}(X) \epsilon^{i j} \partial_{i} X^{\mu} \partial_{j} X^{\nu}+\phi(X)^{(2)} R+\right. \\
\left.+\frac{1}{\alpha^{\prime}} F_{\mu \nu \rho \sigma}(X) h^{i j} h^{k l} \partial_{i} X^{\mu} \partial_{j} X^{\nu} \partial_{k} X^{\rho} \partial_{l} X^{\sigma}+\ldots\right]
\end{gathered}
$$

where together with the metric, dilaton and antisymmetric field, we have displayed an example of a field at the next mass level. Once we include massive fields at the first excited level, the whole tower of massive fields must be included, since they are associated with non-renormalizable interactions from the point of view of the 2-dimensional theory, and all possible terms are in principle generated by the sigma model loop (i.e. $\alpha^{\prime}$ ) expansion.

However, when we compute higher loops in the beta functionals of the massless modes, the massive modes play no role: the operators associated with massive modes are nonrenormalizable and do not mix with the massless sector. More generally, to renormalize background fields at a given mass level, we need only fields at the same or lower mass level [23].

However, since in string theory we have an infinite tower of massive modes, while the effective low energy action is written in terms of massless fields only, it is clear that we have somehow integrated over the massive modes. This physical interpretation is confirmed by the computation of the effective action through the comparison with $S$-matrix elements. In this case the effective action, at all orders in $\alpha^{\prime}$ but without $e^{\phi}$ corrections, is obtained by requiring that it reproduces the string amplitudes at genus zero, with an arbitrary number of insertions of vertex operators for the massless fields. In the field theory language, we expect that the $\alpha^{\prime}$ corrections to the amplitudes can be represented as a sum of tree level exchange graphs with massive string modes in the intermediate state. This is indeed the case, as can be read from the computation of Gross and Witten [14] of graviton scattering in type II superstring theory. In this case the tree amplitude is [24, 14]

$$
A=\frac{G_{N}^{2}}{128}\left[\frac{\Gamma(-s / 8) \Gamma(-t / 8) \Gamma(-u / 8)}{\Gamma\left(1+\frac{1}{8} s\right) \Gamma\left(1+\frac{1}{8} t\right) \Gamma\left(1+\frac{1}{8} u\right)}\right] K\left(\epsilon^{(i)}, k^{(j)}\right) \tilde{K}\left(\epsilon^{(i)}, k^{(j)}\right),
$$


where $G_{N}$ is the gravitational constant, $s, t, u$ the Mandelstam variables and $K, \tilde{K}$ are kinematical factors.

Expanding the term in brackets for small momenta, one gets a leading term $-2^{9} /(s t u)$, which, when inserted back in eq. (3.2), gives the tree level scattering amplitude derived from the lowest order effective action; and a correction term $-2 \zeta(3)$, which gives the leading correction to the effective action; from the kinematical factors $K, \tilde{K}$ one finds that it corresponds to a term in the effective action quartic in the Riemann tensor [14] (remember that for type II superstrings the coefficient $k$ in eq. (2.1) is zero, i.e. there is no $\sim R_{\mu \nu \rho \sigma}^{2}$ correction).

Writing the Riemann zeta function as $\zeta(3)=\sum_{n=1}^{\infty} n^{-3}$, we see that the correction can be interpreted as a sum over an infinite tower of intermediate states, and that states at level $n$ give a contribution $\sim 1 / n^{3}$.

This means that the $\alpha^{\prime}$ corrections are dominated by the first few massive string states.

For instance, summing over the first 10 excited states, we get $\sum_{n=1}^{10} 1 / n^{3} \simeq 1.1975 \ldots$, to be compared with the value of the Riemann zeta function $\zeta(3) \simeq 1.2020 \ldots$.

Having obtained a physical picture of the mechanism that is responsible for the perturbative $\alpha^{\prime}$ corrections to the effective action (integration over the first few massive modes), we proceed in the next section to discuss the physical mechanism that is responsible for the regularization of the singularity.

\subsection{Small distances vs. large energy singularities}

It can be useful to distinguish between small distance singularities and large energy singularities. In field theory small distances means large energies, and the distinction is therefore meaningless. In string theory, however, the behavior of amplitudes in high energy scattering suggests the existence of a generalized uncertainty principle of the form 25]

$$
\Delta x \geq \frac{1}{\Delta p}+\text { const. } G_{N} \Delta p .
$$

(Such an uncertainty principle is also suggested by general properties of quantum black holes [26].) Therefore in string theory the connection between high energies and small distances is not completely trivial and it is useful to consider the two cases separately.

The most basic reason why we expect strings to regulate singularities at small distances is that the very notion of invariant point-like event is not meaningful in string theory. While 
in quantum field theory an invariant event can be defined through the splitting of a particle in two, no such concept exist for strings, since the point in space-time where a string splits in two depends on the Lorentz frame used [27].

At large energies, the regulating mechanism is instead the exponential growth of the density of states. The asymptotic density of states has the form

$$
d(M) \sim\left(\frac{M}{M_{0}}\right)^{-b} e^{M / M_{0}},
$$

where $M_{0}=c / \sqrt{\alpha^{\prime}}$ and $b, c$ are numerical constants, which depend on the string theory under consideration. An example of how the density of states prevents quantities with dimension of energy from growing arbitrarily large is given by the fact that we cannot raise the temperature of a system beyond the Hagedorn temperature, $T_{\mathrm{HAG}}=M_{0}$, because otherwise the canonical partition function diverges.

Another interesting example is given by the existence of a limiting value of the electric field for an open bosonic string. In the presence of an external electric field $E$, the rate for charged-string pair production is in fact 28]

$$
w \sim \sum_{S} q_{S} \sum_{k=1}^{\infty}(-1)^{k}\left(\frac{|\epsilon|}{k}\right)^{(d+1) / 2} \exp \left\{-\frac{\pi k}{|\epsilon|}\left(M_{S}^{2}+\epsilon^{2}\right)\right\} .
$$

The sum goes over all physical string states $S$, with mass $M_{S}$, and $q_{S}$ is a factor that depends on the charge of the state $S$; in the weak field limit, $\epsilon \simeq e E+o\left(E^{3}\right)$, where $e$ is the total electric charge of the string, and one recovers the Schwinger result. As long as $\epsilon$ is finite, the factor $\exp \left(-\pi k M_{S}^{2} /|\epsilon|\right)$ ensures the convergence of the rate, even if the sum over the states $S$, for large masses, is an integral with the exponentially growing density of states (3.4). However, at a critical value of the electric field, $\epsilon$ goes to infinity and the rate therefore diverges.

The cosmological singularity is a large energy singularity: the Hubble constant, and therefore the curvature or the energy density, diverges on the lowest order solutions of the equations of motion. We therefore expect that the regulating mechanism has to do with the existence of an infinite set of massive states; in fact, we expect something similar to what happens in eq. (3.5), since the existence of a maximum electric field and of a maximum gravitational field do not seem to be two fundamentally different problems.

So, the first conclusion is that we should not necessarily expect that perturbative $\alpha^{\prime}$ corrections regulate the singularity. As we discussed in sect. 3.1, these corrections can be 
accounted for with an accuracy better than one per cent summing over the first ten mass levels. The regularization of the singularity, instead, must be crucially related to the existence of an infinite tower of massive states. Of course, this does not mean that the mechanism proposed in [7] cannot regulate the singularity. It simply means that we do not have any $a$ priori argument that allows us to confidently state that the mechanism will operate.

The second point suggested by the above considerations is to look for massive modes production in a gravitational field. This will be discussed in the next section.

\section{Production of highly excited modes}

A time-varying gravitational field produces particles. The general mechanism is the existence of a non-trivial Bogoliubov transformation between the in and the out vacuum. For instance, in De Sitter space with Hubble constant $H$, a detector moving on a geodetic sees a thermal bath of particles at a temperature

$$
T(H)=\frac{H}{2 \pi} .
$$

This suggests that in De Sitter space a massive string mode with mass $M$ is created with a probability proportional to $\exp (-M / T(H))$. One might wonder whether a truly stringy computation of massive modes creation could give a different answer from the field theory

result (4.1). However, Lawrence and Martinec [29] (see also [30]) used string field theory to compute massive modes production in a FRW space with

$$
d s^{2}=C(\eta)\left(d \eta^{2}-d \vec{x}^{2}\right), \quad C(\eta)=A+B \tanh (\rho \eta)
$$

and they obtained the same result that holds in this model in the field theory limit, discussed e.g. in ref. [31.

The above result suggests that the rate of energy density production due to massive modes creation during the expansion is approximately

$$
\begin{aligned}
\dot{\rho} & \simeq \frac{1}{\lambda_{s}^{d}} \int_{M_{0}}^{\infty} d M M d(M) e^{-2 \pi M / H} \sim \\
& \sim \frac{M_{0}^{2}}{\lambda_{s}^{d}} \int_{1}^{\infty} d x x^{1-b} e^{-\gamma x},
\end{aligned}
$$

where

$$
\gamma=\frac{2 \pi M_{0}}{H}-1=\frac{2 \pi c}{H \sqrt{\alpha^{\prime}}}-1
$$


and $d(M)$ is the density of states, given asymptotically by eq. (3.4). This formula, which has been suggested in [29] as valid for all the mass levels, is also qualitatively confirmed by the explicit computation of the Bogoliubov coefficients for the graviton production, i.e. at mass level zero [2, 32]. In this case, in fact, one finds that $\left|\beta_{k}\right|^{2}$ is a number of order 1 for energies of the order of the string mass (red-shifthing the frequency at the present epoch, this means that $\left|\beta_{k}\right|^{2}=O(1)$ if the physical frequency, as seen today, is of order $10 \mathrm{GHz}$ ), and has an exponential cutoff for larger frequencies. Actually, the spectrum also shows some deviations from an exact black body spectrum (it goes like $\omega^{3}$ for low frequencies but as $\omega^{3-2 \mu}$, where $\mu$ depends on details of the string phase, for intermediate frequencies.) These deviations, however, are not very relevant to the present discussion.

Equation (4.3) clearly has a regularizing effect on the growth of $H$. When $H$ exceeds a critical value $H_{c}$, given by $H_{c} /(2 \pi)=M_{0}$, the production rate diverges. This result can also be interpreted by noting that $H /(2 \pi)$ is the Hawking temperature in De Sitter space, and therefore the condition on $H_{c}$ is the requirement that the Hawking temperature does not exceed the Hagedorn temperature, $T_{\mathrm{HAG}}=M_{0}$.

The effect that we are discussing is non-perturbative in $\alpha^{\prime}$. In fact, for $\gamma>0$ the integral in eq. (4.3) is an incomplete gamma function, and from its known asymptotic expansion we find that, in the limit $\alpha^{\prime} \rightarrow 0$,

$$
\dot{\rho} \sim\left(\frac{1}{\alpha^{\prime}}\right)^{\frac{d}{2}+1} \exp \left\{-\frac{2 \pi c}{H \sqrt{\alpha^{\prime}}}\right\},
$$

and it is therefore non-analytic in $\alpha^{\prime}$ at $\alpha^{\prime}=0$.

To understand in more detail the evolution of the cosmological model, we tentatively use the expression (4.3) for the energy density produced, as a backreaction term in the cosmological equations. The equations of motion, without perturbative $\alpha^{\prime}$ corrections, and including matter sources with $T_{\mu}^{\nu}=\left(\rho(t),-\delta_{i}^{j} p(t)\right)$, are [2, 5] (in the isotropic case, in $d$ spatial dimensions)

$$
\begin{gathered}
\dot{\bar{\phi}}^{2}-d H^{2}=2 \lambda_{s}^{d-1} e^{\bar{\phi}} \bar{\rho} \\
2 \ddot{\bar{\phi}}-\dot{\bar{\phi}}^{2}-d H^{2}=0
\end{gathered}
$$

\footnotetext{
${ }^{4}$ In an expanding Universe one should also add to $\dot{\rho}$ a term $-a H \rho$ representing the dilution of string density due to the expansion [33], and $a=d-1$ for long strings. However, this term is not very important for the qualitative discussion presented below, as we checked also numerically.
} 


$$
\dot{H}-H \dot{\bar{\phi}}=\lambda_{s}^{d-1} e^{\bar{\phi}} \bar{p}
$$

where $\bar{\phi}=\phi-d \log a, e^{\bar{\phi}} \bar{\rho}=e^{\phi} \rho, e^{\bar{\phi}} \bar{p}=e^{\phi} p$. We use eq. (4.3) for the time derivative of $\rho$; the pressure $p$ is determined by the conservation of the energy momentum tensor, which gives

$$
\dot{\rho}+d H(\rho+p)=0 .
$$

This is equivalent to requiring that the constraint equation (4.6) is conserved by the dynamical equations of motion (4.7) and (4.8). Since we know $\dot{\rho}$ rather than $\rho, p$, these are in principle integro-differential equations. However, we can combine eq. (4.8) and eq. (4.6) so that only the combination $\rho+p$ appears, which is expressed through $\dot{\rho}$ using eq. (4.9), and we are left with ordinary differential equations. Writing everything in terms of $\phi$ rather than $\bar{\phi}$, the dynamical equations of motion can then be written as

$$
\begin{gathered}
2 \dot{H}+\dot{\phi}^{2}-2(d+1) H \dot{\phi}+d(d+1) H^{2}=-\frac{2}{d} \lambda_{s}^{d-1} e^{\phi} \frac{\dot{\rho}(H)}{H}, \\
2 \ddot{\phi}-2 d \dot{H}-\dot{\phi}^{2}+2 d H \dot{\phi}-d(d+1) H^{2}=0 .
\end{gathered}
$$

The constraint equation

$$
\dot{\phi}^{2}+d(d-1) H^{2}-2 d H \dot{\phi}=2 \lambda_{s}^{d-1} e^{\phi} \rho
$$

is still an integro-differential equation. However, we impose the constraint at the initial time $t_{0} \ll-1$ (in units $\lambda_{s}=1$ ), when the right-hand side is negligible. The constraint is then automatically preserved by the evolution (we use its conservation as a check of the numerical integration). We have then integrated these equations numerically. The results are qualitatively the same for any $d$ and any value of $b, c$ in eqs. (4.3) and (4.4) that we have tried. The plots that we present refer to $d=3$ and to the values $b=10, c=10$ (this value of $c$ is responsible for the values $\sim O(10)$ of the $H$-scale in Fig. 1). Similar results holds for $c=[(2+\sqrt{2}) \pi]^{-1} \simeq 0.09$, which is the value for the heterotic string.

Figure 1 shows the behavior of the Hubble parameter versus cosmic time $t$. At large negative values of $t$ we recover of course the lowest order solution, since the production of massive string states is exponentially small; $H(t)$ then reaches a maximum value and then very abruptly bounces off and goes to zero at a finite value of $t$. At this point the numerical integration stops. The behavior of $\dot{\phi}$ is shown in fig. 2, while fig. 3 shows $\dot{\rho}(t)$.

\footnotetext{
${ }^{5}$ The integration stops because the integral in eq. (4.3) is only defined for $\gamma>0$. For negative values of
} 
These plots show that some important aspects of the regularization mechanism are still missing from our equations. In fact, even if $H$ does not diverge, $\dot{H}$ becomes very large when $H$ goes to zero, and the Ricci scalar $\mathcal{R}=-6\left(\dot{H}+2 H^{2}\right)$ in this regime is dominated by $\dot{H}$ and is large. The missing ingredient can be traced back to eq. (4.1). This form of the temperature holds for De Sitter space, where $H$ is constant and $\dot{H}=0$. It is inappropriate at a stage of the cosmological evolution when $H$ is small compared to the critical value but $\dot{H}$ is large. Indeed, at this stage our equations predict no massive mode creation, while a large $\dot{H}$, as any strong time-varying gravitational field, will produce particles, much the same as a large $H$. This is quite clear physically, but it is anyway interesting to check this point in a toy model. One can consider for instance a model with a scale factor such that $H=A / \cosh (\omega t)$, with $A, \omega>0$. If $A \ll 1$ but $A \omega \gg 1$ the Hubble constant is always small during the evolution while $\dot{H}$ reaches a maximum value $\dot{H}_{\max }=A \omega / \sqrt{2} \gg 1$. A straightforward computation gives a production rate of massive particles $\sim \exp (-M / T)$ with $T \simeq \dot{H}_{\max }$.

So, at least at the level of phenomenological description of the string phase, one should generalize eq. (4.1) to

$$
T=T(H, \dot{H}, \ddot{H}, \ldots, \dot{\phi}, \ddot{\phi}, \ldots),
$$

where all higher order derivatives of $H$ appear. Note that we also included a dependence on $\dot{\phi}$ because, changing frame, say from the string to the Einstein frame, the derivative of the dilaton field contributes to the gravitational field, and therefore in a generic frame even $\dot{\phi}$ must contribute to massive modes production. For physical reasons, we expect that $T$ will be a sum of positive definite contributions. For instance, a possible plausible form could be

$$
T=\frac{1}{2 \pi}\left(H^{2}+c_{1} \dot{H}^{2}+c_{2} \dot{\phi}^{2}+\ldots\right)^{1 / 2}
$$

with $c_{1}, c_{2}>0$. Now it becomes technically impossible to solve the differential equations, since already including a term $\dot{H}$ into $T$, the highest derivative of $H$ in the differential equations appears as argument of the incomplete gamma functions, which makes the equations intractable, even numerically. However, the qualitative effects of eq. (4.10), combined with an expression of the form of eq. (4.13,4.14) for the temperature, can be understood as follows. $\bar{H}$ we could replace $H$ is eq. (4.4) with $|H|$. This is motivated by the fact that, for instance, in the model defined in eq. (4.2) the production rate is the same independently of whether we consider a model with a scale factor increasing in time or decreasing in time with the same rate. However, as we will now discuss, a more crucial modification of our equations is needed. 
When any of the derivative of $H$ or $\phi$ exceeds a critical value of order one in units $\lambda_{s}=1$, the right-hand side of eq. (4.10) diverges, while the left-hand side is finite. So there can be no solution where $H, \dot{H}, \dot{\phi}, \ldots$ are larger than their critical value. Similarly to what happens to $H$ in fig. 1, when $\dot{H}, \dot{\phi}$, etc. approach their respective critical values, the effect of the term $\dot{\rho}$ in eq. (4.10) is to repel them away. Furthermore, $H$ cannot go to zero. Indeed, unless all higher derivatives $\dot{H}, \dot{\phi}, \ldots$ vanish (as it happens at $t \rightarrow-\infty$ if the evolution starts from the string perturbative vacuum), then $\dot{\rho}(H, \dot{H}, \dot{\phi}, \ldots)$ does not vanish for $H \rightarrow 0$, contrarily to what happens when we use the expression $T(H)=H / 2 \pi$, see eq. (4.5). Therefore the right-hand side of eq. (4.10) diverges if $H \rightarrow 0$ while the left-hand side is finite, because $\dot{H}, \dot{\phi}$ are bounded. Therefore, there can be no solution with $H$ approaching zero. So, we expect a solution in which the scale factor in the string frame is always expanding, $H>0$, possibly in a very complicated way, but still without divergences in the curvature or in any other quantity. p

The results obtained trying to incorporate massive mode production as a backreaction on the metric and dilaton field show that massive modes may have a very important effect on the evolution of the cosmological model. However, the very complexity of the equations that describe the backreaction of massive modes on the massless modes $\phi, g_{\mu \nu}$ indicates that a treatement as backreaction is not appropriate. If we enter a full stringy regime, with an infinite tower of excited massive fields, it is not surprising that a description of the cosmological model in terms of classical evolution of massless fields runs into difficulties.

Finally we stress that, even if our results allow to obtain some understanding of the physical mechanism that regularizes the singularity of the lowest order solution, still this mechanism cannot be the full story, since it does not provide a solution that, besides being possibly non-singular, is also smoothly connected with the present radiation dominated phase. This is the so-called graceful exit problem [8, 9, 10, 11]. It can be posed as follows. From eq. (4.6) one sees that there are two solutions for $\dot{\bar{\phi}}$, given by

$$
\dot{\bar{\phi}}= \pm\left(d H^{2}+2 \lambda_{s}^{d-1} e^{\phi} \rho\right)^{1 / 2}
$$

These two solutions are usually referred to as the $(+)$ and $(-)$ branches, depending on the

\footnotetext{
${ }^{6}$ We stress that this is not a proof that there will be a non-singular solution. In principle it may happen that at some value of $t$ two different solutions 'collide' and move into the complex plane, as we have seen in sect. 2.3 for the equation with perturbative $\alpha^{\prime}$ corrections.
} 
choice of sign in front of the square root. The pre-big-bang solution starts on the $(+)$ branch; this ensures that the solution start from a regime of low curvature and couplings, and goes through a phase of inflation driven by the kinetic energy of the dilaton field. The final state, however, should correspond to a radiation dominated FRW model with constant dilaton,

and therefore $\dot{\bar{\phi}}=\dot{\phi}-d H<0$, and we are on the $(-)$ branch. Therefore at least one branch change must take place. Eq. (4.15) shows that a necessary condition for this to happen is that, at some point in the evolution, the matter density $\rho$, in the string frame, be negative. Further global conditions are necessary to ensure that no further branch change back to the $(+)$ branch takes place, and have been discussed in refs. [10, 11]. However, we see that, if we include massive modes as the only source of energy density, $\rho$ is necessarily positive and no branch change can occur. Other source of corrections, like $\alpha^{\prime}$ correction, provide an effective contribution to $\rho$ which is negative and therefore allows branch changes. Still, to satisfy the global conditions for a succesful exit completion, $e^{\phi}$ corrections must also be included. An example in which the combined effect of $\alpha^{\prime}$ corrections and $e^{\phi}$ corrections provides a successful exit has been discussed in [1]. So, it is clear that the effect that we have studied in this paper is only an ingredient of the mechanism that finally can produce a well-motivated and non-singular cosmological model, that eventually matches standard cosmology.

\section{Conclusions}

The lowest-order cosmological solutions obtained from the string effective action are singular, and therefore corrections to the lowest order action must play a crucial role. In string theory there are two kind of corrections, 'stringy corrections', parametrized by $\alpha^{\prime}$, and loop corrections, parametrized by $e^{\phi}$. In this paper we have drawn attention to the fact that $\alpha^{\prime}$ corrections come into two types, perturbative and non-perturbative, and that they have a distinct physical origin. Perturbative corrections are related to the integration over the first few massive modes while non-perturbative corrections comes from the exponentially growing density of massive states. As we discussed in sect. 3, this physical interpretation makes clear that non-perturbative $\alpha^{\prime}$ corrections are expected to have a regularizing effect.

The explicit verification of the regularization mechanism meets difficulties both for pertubative and non-perturbative $\alpha^{\prime}$ corrections. In the former case, a truncation at any finite order in perturbation theory gives scheme-dependent results, so that an all-order computa- 
tion is in principle required; since an all-order perturbative computation is anyway faced with the problem of the convergence and resummation of the asymptotic series, this is already a hint of the fact that the full answer requires the inclusion of non-perturbative effects.

In the case of non-perturbative corrections related to massive modes production, the technical difficulty that we have to face is the inclusion of the effect of all higher order derivative of $H$ and $\phi$ in the computation of the production rate of massive modes. However, independently of the technical difficulties, the mechanism has a clear physical interpretation, which suggests that it regularizes the lowest order solution.

We conclude by pointing out briefly a possible phenomenological consequence of the scenario in which in the string phase we have highly excited string modes. The massive string states produced will eventually decay. The decay of the excited string levels has been studied in ref. [34. The result is that, for large level number $N$, the width is dominated by decays in which one of the products is massless, i.e. by transitions of the form $N \rightarrow N-1$ with emission of a graviton or another massless particle. From the mass formula $M_{N}^{2} \sim N$, the level spacing is $\Delta M_{N} \sim 1 / M_{N}$. If a string at an excited level $N$ decays at level $N-1$ with emission of a graviton of frequency $\omega=\Delta M_{N}$, we get transitions at the frequencies $f_{N}=f_{0} / \sqrt{N}$. The most naive estimate of the frequency $f_{0}$, as seen today, is obtained by redshifting the string mass from a string time to the present epoch, which gives $f_{0}=\omega_{\text {today }} /(2 \pi)$ of the order of $10-10^{2} \mathrm{GHz}$, which is also the typical cutoff value of the gravitons produced by quantum vacuum fluctuations [2, 32, 35]. However, this radiation is produced within the string phase, rather than at the transition between the string phase and the $F R W$ phase, and a further red-shift must be included, to take into account the expansion from the time of production to the onset of a standard FRW cosmology. We cannot compute it, in the absence of a knowledge of the mechanism that terminates the string phase. There is, however, the interesting possibility that, because of this further redshift and/or the factor $1 / \sqrt{N}$ in $f_{N}$, this gravitational radiation might be shifted in the frequency band accessible to the LIGO/Virgo gravitational wave detectors, which are expected to operate in the $6 \mathrm{~Hz}-1 \mathrm{kHz}$ region.

Concerning the intensity of this gravitational radiation, it can be estimated as follows. If there is not a subsequenty inflationary phase below the string phase, also the photons that we observe today in the CMBR have been produced during the large curvature phase 
of the model, by massive modes decay. Since at this scale the rates for photon and graviton production are comparable, this suggests that the overall intensity of the relic gravitational wave background produced by the decay of massive string modes has an energy density comparable to the energy density of the $2.7 \mathrm{~K}$ radiation.

\section{Acknowledgements}

I am grateful to Alessandra Buonanno, Stefano Foffa, Krzysztof Meissner, Riccardo Sturani, Carlo Ungarelli and Gabriele Veneziano for very useful discussions. I am especially grateful to Jorge Russo for invaluable discussions. I also thank the referee for stimulating comments. 


\section{References}

[1] G. Veneziano, Phys. Lett. B265 (1991) 287.

[2] M. Gasperini and G. Veneziano, Astropart. Phys. 1 (1993) 317; Mod. Phys. Lett. A8 (1993) 3701; Phys. Rev. D50 (1994) 2519.

[3] G. Veneziano, in "String Gravity and Physics at the Planck Energy Scale", N. Sanchez and A. Zichichi eds., Kluwer Publ., Dordrecht, 1996, pg. 285; M. Gasperini, ibid. pg. 305.

An up-to-date collection of references on string cosmology can be found at http://www.to.infn.it/teorici/gasperini/

[4] R. Brandenberger and C. Vafa, Nucl. Phys. B316 (1989) 391;

B. Campbell, A. Linde and K. Olive, Nucl. Phys. B355 (1991) 146;

E. Copeland, A. Lahiri and D. Wands, Phys. Rev. D50 (1994) 4868;

I. Antoniadis, J. Rizos and K. Tamvakis, Nucl. Phys. B415 (1994) 497.

[5] A. Tseytlin and C. Vafa, Nucl. Phys. B372 (1992) 443.

[6] K.A. Meissner and G. Veneziano, Phys. Lett. B267 (1991) 33; Mod. Phys. Lett. A6 (1991) 3397;

M. Gasperini and G. Veneziano, Phys. Lett. B277 (1992) 256;

A. Tseytlin, Mod. Phys. Lett. A6 (1991) 1721;

A. Sen, Phys. Lett. B271 (1991) 295.

[7] M. Gasperini, M. Maggiore and G. Veneziano, Nucl. Phys. B494 (1997) 315.

[8] R. Brustein and G. Veneziano, Phys. Lett. B329 (1994) 429; N. Kaloper, R. Madden and K. Olive, Nucl. Phys. B452 (1995) 677.

[9] M. Gasperini, J. Maharana and G. Veneziano, Nucl. Phys. B472 (1996) 349;

S. Rey, Phys. Rev. Lett. 77 (1996) 1929; hep-th/9609115;

M. Gasperini and G. Veneziano, Phys. Lett. B387 (1996) 715; 
A. Buonanno, M. Gasperini, M. Maggiore and C. Ungarelli, Class. Quant. Grav. 14 (1997) L97;

[10] R. Brustein and R. Madden, Phys. Lett. B410 (1997) 110.

[11] R. Brustein and R. Madden, Phys. Rev. D57 (1998) 712.

[12] C. Callan, D. Friedan, E. Martinec and M. Perry, Nucl. Phys. B262 (1985) 593.

[13] E. Fradkin and A. Tseytlin, Phys. Lett. B158 (1985) 316; Nucl. Phys. B261 (1985) 1.

[14] D. Gross and E. Witten, Nucl. Phys. B277 (1986) 1.

[15] B. Zwiebach, Phys. Lett. B156 (1985) 315.

[16] R. Metsaev and A. Tseytlin, Nucl. Phys. B293 (1987) 385.

[17] S. Deser and A. Redlich, Phys. Lett. B176 (1986) 350;

[18] K. Förger, B. Ovrut, S. Theisen and D. Waldram, Phys. Lett. B388 (1996) 512.

[19] K. Meissner, Phys. Lett. B392 (1997) 298.

[20] N. Kaloper and K. Meissner, Phys. Rev. D56 (1997) 7940.

[21] R. Brustein, D. Nemeschansky and S. Yankielowicz, Nucl. Phys. B301 (1988) 224.

[22] A.A. Tseytlin, Phys. Lett. B185 (1987) 59.

[23] I.L. Buchbinder, E.S. Fradkin, S.L. Lyakhovich and V.D. Pershin, Phys. Lett. B304 (1993) 239.

[24] M. Green and J. Schwarz, Nucl. Phys. B181 (1981) 502, B198 (1982) 441.

[25] G. Veneziano, Europhys. Lett. 2 (1986) 199;

D. Amati, M. Ciafaloni and G. Veneziano, Phys. Lett B216 (1989) 41, B197 (1987) 81;

Int. J. Mod. Phys. A3 (1988) 1615; Nucl. Phys. B347 (1990) 530;

D.J. Gross and P.F. Mende, Phys. Lett. B197 (1987) 129; Nucl. Phys. B303 (1988) 407;

K. Konishi, G. Paffuti and P. Provero, Phys. Lett. B234 (1990) 276. 
[26] M. Maggiore, Phys. Lett. B304 (1993) 65.

[27] M. Green, J. Schwarz and E. Witten, Superstring Theory, Cambridge Univ. Press, Cambridge, 1987, Vol. 1, pg. 29.

[28] C. Bachas and M. Porrati, Phys. Lett. B296 (1992) 77.

[29] A. Lawrence and E. Martinec, Class. Quantum Grav. 13 (1996) 63.

[30] E. Martinec, Class. Quant. Grav. 12 (1995) 941.

[31] N. Birrel and P.C.W. Davies, Quantum fields in curved space, Cambridge University Press, Cambridge 1982.

[32] R. Brustein, M. Gasperini, M. Giovannini and G. Veneziano, Phys. Lett B361 (1995) 45 ;

R. Brustein, M. Gasperini and G. Veneziano, Phys. Rev. D55 (1997) 3882;

A. Buonanno, M. Maggiore and C. Ungarelli, Phys. Rev. D55 (1997) 3330;

[33] N. Turok, Phys. Rev. Lett. 60 (1988) 549.

[34] R.B. Wilkinson, N. Turok and D. Mitchell, Nucl. Phys. B332 (1990) 131.

[35] B. Allen and R. Brustein, Phys. Rev. D55 (1997) 3260. 


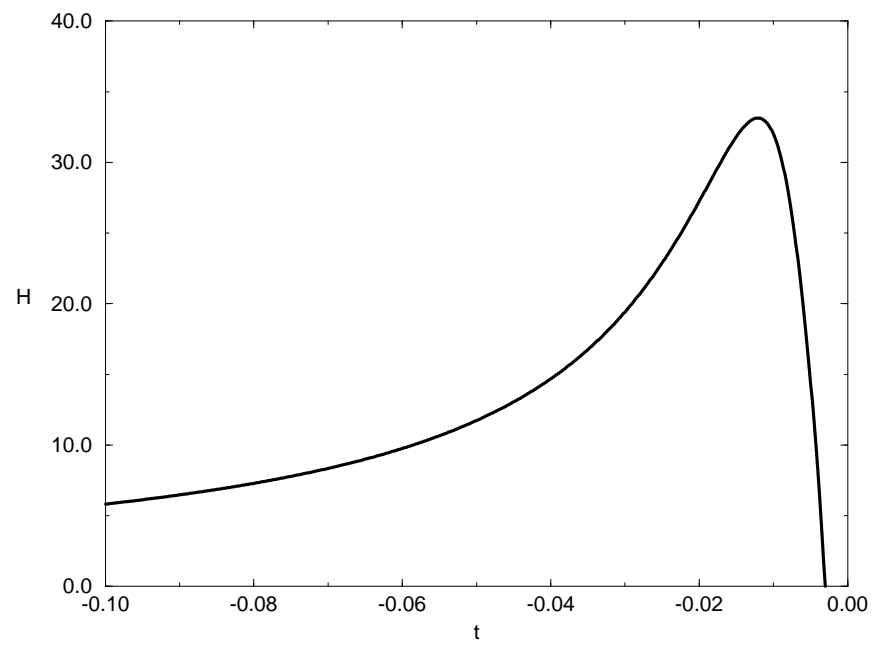

Figure 1: The Hubble parameter $H$ as a function of time. The initial conditions for the numerical integration are $t_{0}=-20, \phi\left(t_{0}\right)=-10, H\left(t_{0}\right)=-1 /\left(t_{0} \sqrt{3}\right) ; \dot{\phi}\left(t_{0}\right)$ is determined by the constraint equation.

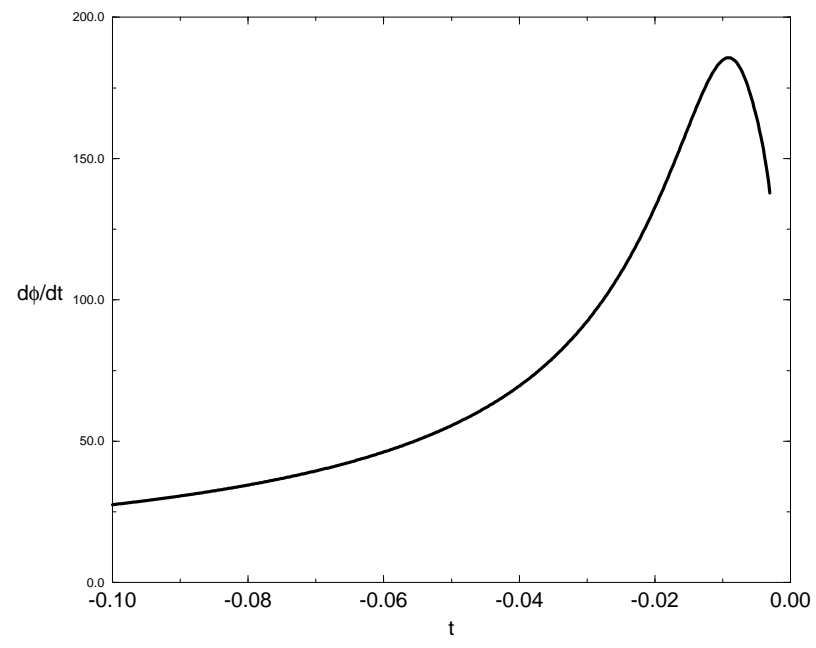

Figure 2: $\dot{\phi}$ as a function of time. Same initial conditions as in fig. 1. The integration stops when $H$ reaches zero. 


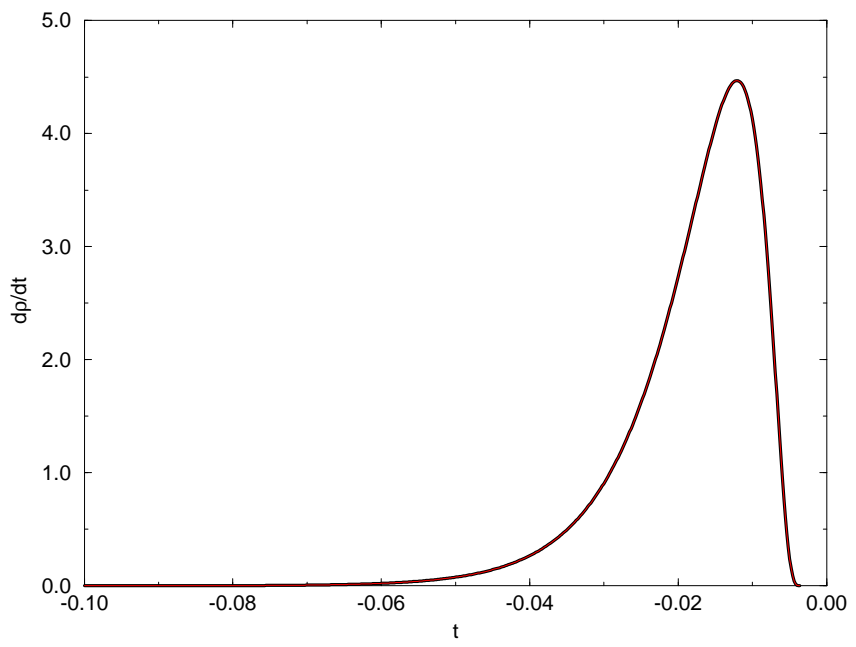

Figure 3: $\dot{\rho}$ as a function of time. Same initial conditions as in fig. 1 . 